\title{
Severity of hypoxia modulates effect of CPAP on myocardial stress as measured by highly sensitive troponin $\mathrm{T}$
}

\author{
Amir Sharafkhaneh ${ }^{1,3^{*}}$ D, Jennifer Katigbak ${ }^{4}$, Max Hirshkowitz ${ }^{1,3}$, Hossein Sharafkhaneh $^{3}$, Saba P. Sharafkhaneh ${ }^{5}$, \\ Christie M. Ballantyne ${ }^{3,6}$, Biykem Bozkurt ${ }^{2,3}$ and Vijay Nambi ${ }^{2,3,6}$
}

\section{Dear editor}

Obstructive sleep apnea (OSA) is associated with increased risk for cardio- and cerebrovascular diseases $[1,2]$. Animal studies propose intermittent hypoxia and the resulting inappropriate activation of sympathetic nervous system $[3,4]$ as the major link between OSA and cardio- and cerebrovascular comorbidities. However, the OSA severity level at which continuous positive airway pressure (CPAP) therapy offers cardio- and cerebrovascular benefit (reduction in indices of myocardial stress/injury) is a matter of debate. Sub-clinical injury to the myocardium is considered to be a precursor for the development of incident cardiovascular disease including heart failure [5]. Troponin $T$ measured with a high sensitivity assay (Hs-TnT) has been shown to predict incident heart failure and cardiovascular death [6]. Further, increase or decrease in Hs-TnT over time predicted increased or decreased incidence of heart failure and cardiovascular death $[7,8]$. Hs-TnT has also shown to be increased with presence and severity of OSA [9]. Stronger correlations were reported with nadir $\mathrm{SpO} 2$ [10]. Roca and colleagues, in a cohort of 1645 subjects free of coronary artery disease and heart failure with a median follow up of 12.4 years, reported that hs-TnT was associated with risk of death or incident heart failure across various categories of OSA after adjusting for 17 potential confounders [11]. Surprisingly, a recent study showed increased Hs-TnT after 12 months of CPAP therapy in patients with OSA. However, the utilization of CPAP was not reported [12]. We report changes in Hs-TnT level as a

\footnotetext{
* Correspondence: amirs@bcm.edu

${ }^{1}$ Section of Pulmonary, Critical Care and Sleep Medicine, Medical Care Line, Michael E. DeBakey Veterans Affairs Medical Center, MED VA Medical Center, Bldg. 100 (111i), 2002 Holcombe Blvd, Houston, TX 77025, USA

3 Department of Medicine, One Baylor Plaza, Houston, TX 77030, USA

Full list of author information is available at the end of the article
}

marker of myocardial stress/injury in a cohort of CPAP compliant patients with OSA.

\section{Methods}

This study was approved by institutional review board of Baylor College of Medicine and Research \& Development Committee of Michael E. DeBakey VA Medical Center. All participants signed an informed consent form. We enrolled adult subjects with confirmed OSA (apnea + hypopnea index (AHI) of $\geq 15$ obstructive and/ or mixed events/h) using attended polysomnography (PSG). Subjects qualifying for the PSG underwent blood sampling and completed an Epworth Sleepiness Scale (ESS). The participants underwent attended CPAP titration and were placed on therapy. The best pressure was the one associated with the lowest AHI while the patient slept 20 min, or more. After titration, subjects received a CPAP machine and related accessories (Respironics, REMStar Pro) with card reader to monitor the compliance of CPAP and were followed for 6 months. Subjects were seen 2-3 times during the study and CPAP compliance was checked during the visit. CPAP efficacy was rechecked with overnight pulse oximetry at the end of the study. Blood was collected in the morning between 7 and $8 \mathrm{AM}$ in EDTA-containing tubes and kept at $4{ }^{\circ} \mathrm{C}$ during processing at baseline and after 6 months of CPAP use. Aprotinin $(100 \mu \mathrm{L}$ containing 0.6 TIU per $\mathrm{mL}$ of blood) was added to one of the tubes and the samples were then centrifuged at $3000 \mathrm{rpm}$ for $30 \mathrm{~min}$. Hs-TnT concentrations were measured with a novel high sensitivity assay, Elecsys Troponin $\mathrm{T}$ (Roche Diagnostics ${ }^{\circ}$ ), on an automated Cobas e411 analyzer with a limit of measurement of $3 \mathrm{ng} / \mathrm{L}[13]$. 


\section{Results}

Twenty-three subjects were enrolled and 20 subjects completed the study. One subject died unexpectedly at home, from unknown causes. Two subjects were lost to follow up. Baseline and follow samples were available for analysis in 13 and 10 subjects, respectively. The subjects were all male, mean age was 59.7 $(\mathrm{SD} \pm 2)$ years, body mass index $(\mathrm{BMI}) 36.5(\mathrm{SD} \pm 1.8)$ $\mathrm{kg} / \mathrm{m}^{2}$. Baseline polysomnogram showed a total sleep time of $264 \mathrm{~min}$ (SD \pm 121.9 ), sleep efficiency of $80 \%$ (SD $\pm 10.9 \%$ ), latency to sleep onset of $14.3 \mathrm{~min}$ (SD \pm 15.77 ), wake after sleep onset of $48.8 \mathrm{~min}(\mathrm{SD} \pm 34.7$ ); with stage $\mathrm{N} 1$ of $11 \%$ (SD \pm 6.5$)$, N2 of $70 \%$ ( $\mathrm{SD} \pm 14.1$ ), $\mathrm{N} 3$ of $11.8(\mathrm{SD} \pm 10.7)$ and REM of $11.8(\mathrm{SD} \pm 10.7)$. Respiratory parameters at baseline were AHI of $50(\mathrm{SD} \pm 6)$ per hour of sleep, nadir $\mathrm{SpO} 2$ of $77(\mathrm{SD} \pm 3)$, and mean time below $\mathrm{SpO} 2$ of $90 \%$ of $59.37 \mathrm{~min}$ (SD 98.77). Hs$\mathrm{TnT}$ at baseline was elevated and correlated with baseline nadir $\mathrm{SpO} 2(\mathrm{SpO} 2)$ (Correlation Coefficient of 0.889, $\mathrm{p}<0.001$ ), mean time below $\mathrm{SpO} 2$ of 90 \% (Correlation of Coefficient of 0.832, p <0.001) but not with AHI or arousal index (Fig. 1). Subjects used CPAP for $165 \pm 17$ days and $5.3 \pm 0.35 \mathrm{~h} /$ night. ESS decreased with CPAP therapy (14.6 vs 9.1, $p<0.05$ ). Systolic and diastolic blood pressures and heart rate remained unchanged throughout the study period. CPAP effectively reversed hypoxia in all subjects (nadir $\mathrm{SpO} 277$ $\pm 3 \%$ at baseline and $89.3 \pm 3$ post CPAP, $p=0.005$ ) (Table 1). Hs-TnT decreased only in subjects with baseline nadir $\mathrm{SpO} 2$ of $80 \%$ or lower $(n=4)$ (Fig. 2). The absolute and percent changes in Hs-TnT compared to baseline strongly correlated with baseline SpO2 (Correlation Coefficients of 0.835 and 0.6419 respectively, both $\mathrm{p}<0.05)$. The absolute and percent
Table 1 Sleep and metabolic parameters before and after CPAP use

\begin{tabular}{lll}
\hline Pre and Post Treatment Data & & \\
\hline & Baseline & Post-CPAP \\
AHI (episodes/h) & $50 \pm 6$ & - \\
CPAP pressure (cm H2O) & - & $10 \pm 3.2$ \\
CPAP use (days) & - & $165 \pm 17$ \\
CPAP use (Hrs/day) & - & $5.3 \pm 0.35$ \\
ESS & $14.6 \pm 1$ & $9.5 \pm 1^{*}$ \\
Nadir SpO2 (\%) & $77 \pm 3$ & $89.3 \pm 3^{*}$ \\
Mean SpO2 (\%) & $93.2 \pm 0.7$ & $93.8 \pm 0.62$ \\
Systolic blood pressure (mmHg) & $124 \pm 3$ & $129 \pm 4$ \\
Diastolic blood pressure (mmHg) & $76 \pm 2$ & $76 \pm 2$ \\
Heart rate (bpm) & $77 \pm 3$ & $72 \pm 3$ \\
Body weight (Kg) & $108 \pm 5.3$ & $109.6 \pm 5.4^{*}$ \\
BUN (mg/dl) & $14.1 \pm 1.19$ & $14.26 \pm 1.41$ \\
Creatinine (mg/dl) & $0.96 \pm 0.09$ & $1.0 \pm 30.06$ \\
Hs-TnT (micrograms/l) & $0.012 \pm 0.0085$ & $0.011 \pm 0.0035^{*}$
\end{tabular}

AHI Apnea + hypopnea index, ESS Epworth sleepiness scale, $\mathrm{Hs}$-TnT Highly sensitive troponin $\mathrm{T}$

*: Significant differences compared to baseline $(p \leq 0.05)$ appear in bold

changes in Hs-TnT did not correlate with baseline AHI.

\section{Discussion}

Our study, consistent with others, suggests that Hs-TnT as a biomarker of myocardial stress/injury is elevated in patients with OSA [11]. In contrast, the increased HsTnT was associated with severity of hypoxia but not AHI or sleep interruption as measured by arousal index. The CPAP effectively reversed the pathophysiology in

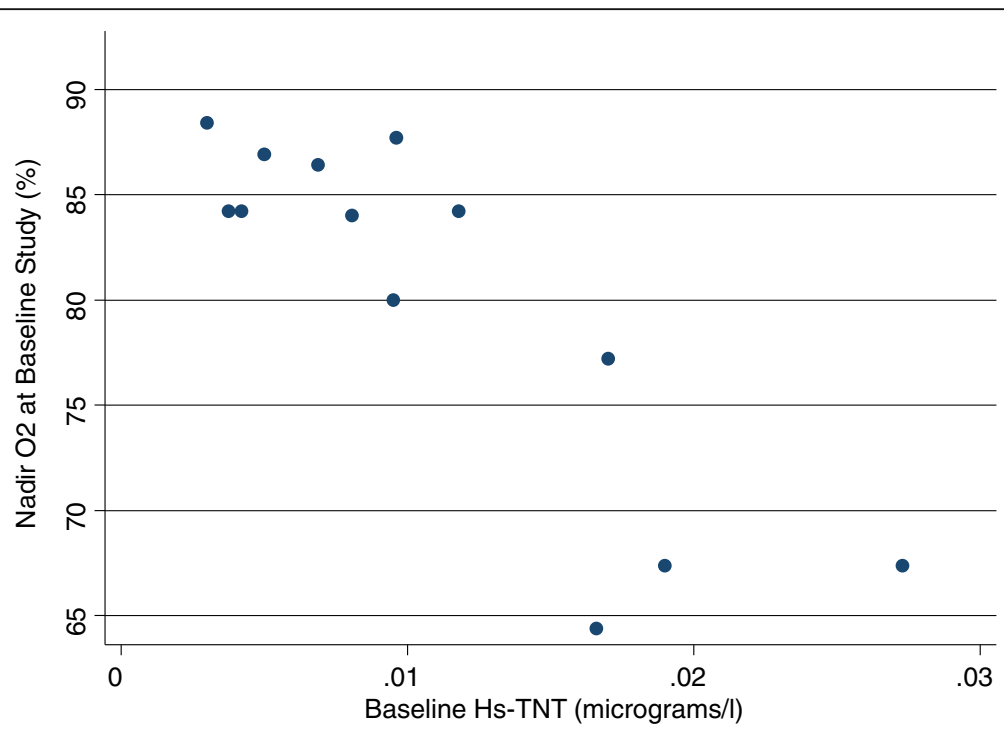

Fig. 1 Baseline Hs-TnT correlates strongly with baseline nadir O2 saturation. Y axis presents the nadir SpO2 during the baseline sleep study 


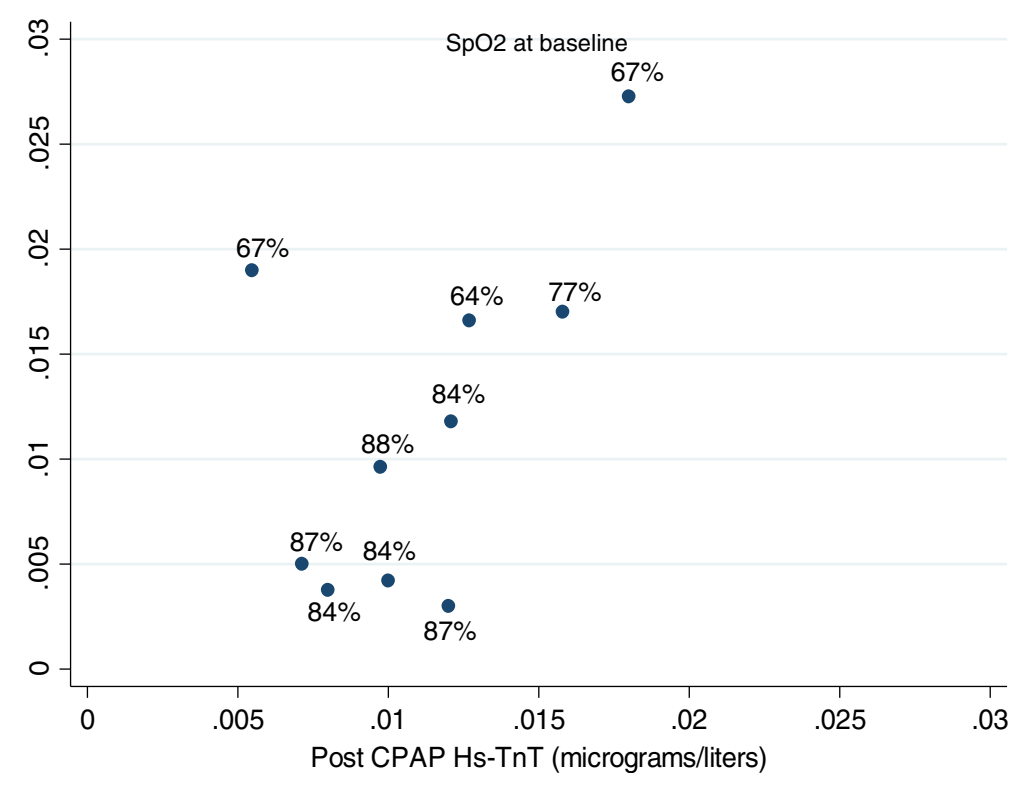

Fig. 2 Follow up versus baseline Hs-TnT: The numbers next to each data points show the nadir SpO2 at baseline

the study subjects but only was associated with decline in Hs-TnT in subjects with more severe hypoxia at baseline. Thus, the data suggests that cardio- and cerbrovascular adverse outcomes related to OSA and the benefits seen with CPAP therapy may greatest to those with pronounced hypoxia as assessed by nadir Sp02 versus other parameters used to assess sleep apnea. A major short coming of our study is the small sample size. While our results are provocative, they must be viewed with caution due to the limited sample size and should be further studied and characterized in larger studies.

\section{Competing interests}

Dr. C.M. Ballantyne and Dr. V. Nambi are co-investigators on a provisional patent filed along with Roche diagnostics for the use of biomarkers in the prediction of heart failure. Dr. C.M. Ballantyne has received research support from Roche diagnostics. Other authors do not report any conflict of interest.

\section{Authors' contribution}

AS designed the study, analyzed the data, conducted statistical analysis and prepared the manuscript. JK analysed the data and prepared the manuscript. $\mathrm{MH}$ designed the study and analysed the data and prepared the manuscript. HS designed the study and analysed the data and prepared the manuscript. SPS analyzed the data and prepared the manuscript. CMB analyzed the data and prepared the manuscript. BB analyzed the data and prepared the manuscript. VN analyzed the data and prepared the manuscript. All authors read and approved the final manuscript.

\section{Acknowledgement}

Roche diagnostics provided reagents and loan of an instrument to conduct the highly sensitive troponin T assay

\section{Author details}

${ }^{1}$ Section of Pulmonary, Critical Care and Sleep Medicine, Medical Care Line, Michael E. DeBakey Veterans Affairs Medical Center, MED VA Medical Center Bldg. 100 (111i), 2002 Holcombe Blvd, Houston, TX 77025, USA. ${ }^{2}$ Section of Cardiology, Michael E. DeBakey Veterans Affairs Medical Center, 2002 Holcombe Blvd, Houston, TX 77025, USA. ${ }^{3}$ Department of Medicine, One
Baylor Plaza, Houston, TX 77030, USA. ${ }^{4}$ University of Houston, Main campus, Houston, TX, USA. ${ }^{5}$ University of Saint Thomas, Houston, TX 77007, USA ${ }^{6}$ Center for Cardiovascular Prevention, Houston Methodist DeBakey Heart and Vascular Center, Houston, TX, USA.

Received: 18 June 2015 Accepted: 3 October 2015

Published online: 16 October 2015

\section{References}

1. Shivalkar B, Van de Heyning C, Kerremans M, Rinkevich D, Verbraecken J, De BW, et al. Obstructive sleep apnea syndrome: more insights on structural and functional cardiac alterations, and the effects of treatment with continuous positive airway pressure. J Am Coll Cardiol. 2006:47:1433-9.

2. Lavie P, Lavie L. Cardiovascular morbidity and mortality in obstructive sleep apnea. Curr Pharm Des. 2008;14:3466-73.

3. Fletcher EC. Sympathetic over activity in the etiology of hypertension of obstructive sleep apnea. Sleep. 2003;26:15-9.

4. Lavie L. Oxidative stress in obstructive sleep apnea and intermittent hypoxia 「Çô Revisited ГÇô The bad ugly and good: Implications to the heart and brain. Sleep Med Rev. 2015;20:27-45.

5. Neeland IJ, Drazner MH, Berry JD, Ayers CR, DeFilippi C, Seliger SL, et al. Biomarkers of chronic cardiac injury and hemodynamic stress identify a malignant phenotype of left ventricular hypertrophy in the general population. J Am Coll Cardiol. 2013;61:187-95.

6. Saunders JT, Nambi V, de Lemos JA, Chambless LE, Virani SS, Boerwinkle E, et al. Cardiac troponin T measured by a highly sensitive assay predicts coronary heart disease, heart failure, and mortality in the Atherosclerosis risk in communities study. Circulation. 2011;123:1367-76.

7. DeFilippi CR, De Lemos JA, Christenson RH. ASsociation of serial measures of cardiac troponin $\mathrm{t}$ using a sensitive assay with incident heart failure and cardiovascular mortality in older adults. JAMA. 2010;304:2494-502.

8. Glick D, DeFilippi CR, Christenson R, Gottdiener JS, Seliger SL. Long-term trajectory of two unique cardiac biomarkers and subsequent left ventricular structural pathology and risk of incident heart failure in community-dwelling older adults at low baseline risk. JACC Heart Fail. 2013;1:353-60.

9. Randby A, Namtvedt SK, Einvik G, Hrubos-Strom H, Hagve TA, Somers VK, et al. Obstructive sleep apnea is associated with increased high-sensitivity cardiac troponin T levels. Chest. 2012;142:639-46. 
10. Hall TS, Herrscher T, Jarolim P, Fagerland MW, Jensen T, Hallen J, et al. Obstructive sleep apnea: no independent association to troponins. Sleep Breath. 2014;18:351-8.

11. Roca GQ, Redline S, Punjabi N, Claggett B, Ballantyne CM, Solomon SD, et al. Sleep apnea is associated with subclinical myocardial injury in the community. The ARIC-SHHS study. Am J Respir Crit Care Med. 2013;188:1460-5

12. Barcelo A, Esquinas C, Bauca JM, Pierola J, de la Pena M, Arque M, et al. Effect of CPAP treatment on plasma high sensitivity troponin levels in patients with obstructive sleep apnea. Respir Med. 2014;108:1060-3.

13. Agarwal SK, Avery CL, Ballantyne CM, Catellier D, Nambi V, Saunders J, et al. Sources of variability in measurements of cardiac troponin $\mathrm{T}$ in a community-based sample: the atherosclerosis risk in communities study. Clin Chem. 2011;57:891-7.

\section{Submit your next manuscript to BioMed Central and take full advantage of:}

- Convenient online submission

- Thorough peer review

- No space constraints or color figure charges

- Immediate publication on acceptance

- Inclusion in PubMed, CAS, Scopus and Google Scholar

- Research which is freely available for redistribution 